\title{
Finite-Time Group Consensus of Distributed Multiagent Systems Based on Competition Mechanism
}

\author{
Yize Yang, ${ }^{1,2}$ Hongyong Yang $\mathbb{D}^{1}{ }^{1}$ and Fei Liu ${ }^{1}$ \\ ${ }^{1}$ School of Information and Electrical Engineering, Ludong University, Yantai 264025, China \\ ${ }^{2}$ School of Electrical Engineering and Telecommunications, The University of New South Wales, Sydney, Australia \\ Correspondence should be addressed to Hongyong Yang; hyyang@yeah.net
}

Received 22 July 2019; Accepted 20 December 2019; Published 16 March 2020

Academic Editor: Emilio Insfran Pelozo

Copyright (c) 2020 Yize Yang et al. This is an open access article distributed under the Creative Commons Attribution License, which permits unrestricted use, distribution, and reproduction in any medium, provided the original work is properly cited.

With the development of communication technology, distributed cooperative controls of multiagent systems (MAS) have been applied in many fields. Based on the communication between the agents, multiple individual systems are composed of a distributed network. Since the function of the agents is different, the distributed network can be divided into many parts. For firstorder/second-order dynamic MAS, group cooperation algorithms with the competition mechanism are proposed in this paper. By applying modern control theory and homogeneous theory, finite-time stabilities for the cooperation motion of dynamic MAS are analyzed. By studying the dynamic properties of agents, finite-time group consensus of distributed MAS based on directed topology is obtained. Finally, the system simulation results are given to illustrate the effectiveness of the conclusion.

\section{Introduction}

As the main branch of distributed systems, multiagent systems (MAS) have been widely concerned by many researchers in the fields of unmanned aerial vehicles, robot control, and distributed sensor networks [1-7]. In the past decade, the study on the distributed control of MAS has attracted great attention and achieved significant research results.

The consensus of MAS requires that multiple agents abide by the communication protocols through the exchange of information between each agent so that all agents in the system can reach the same state. There are a lot of research studies on the consistency problem of MAS [1-7]. In [1], a second-order consensus algorithm for dynamical MAS with leader-following is presented, and the consensus of distributed systems with heterogeneous input delays is studied in the frequency domain. In [2], when the system is affected by exogenous disturbances, the consensus of second-order MAS is studied. Compared with previous results of second-order systems, the consensus of discrete-time third-order MAS in directed networks is investigated in [3]. In $[4,5]$, new distributed protocols are presented to solve the consensus problem for MAS, and the leader-following consensus is achieved by the proposed protocol under both undirected and directed topologies. Different from the consensus of multiagent systems studied in [1-5], containment consensus of fractional-order multiagent systems with sampling control is studied in [6]. [7] also focus on the containment control problem of multiagent systems, and the cooperative control algorithm of compounded fractionalorder multiagent systems is presented.

In the study on the distributed control of MAS, group flocking motion is the significant research direction of MAS. Based on the consensus idea of MAS, the cooperative relationship and the competition mechanism are introduced into dynamic systems, which make the group flocking motion of MAS more suitable for practical application requirements. Supposing the complex network system with multiple subnets, the convergence of the agents in the same subnet is achieved by the communication control protocol, while different subnets converge to different target states. The group controllability of discrete-time MAS with time delay is addressed in [8], where both switching topology and fixed topology are considered. In [9], the group consensus tracking issue of continuous-time second-order MAS under directed fixed topology is studied. The group consensus for 
leaderless MAS with nonidentical dynamics is investigated in [10]. For the network of dynamic agents with switching topologies and communication delays, group consensus problems are discussed in [11]. In [12], a class of dynamic group consensus protocols is proposed for heterogeneous MAS with input time delays, where the dynamic group consensus of heterogeneous MAS is investigated. Different from the communication delay problems [11, 12], the group consensus protocol is designed to compensate for the communication delay actively based on the state prediction scheme in [13].

In order to further meet the requirements of practical engineering, it is especially important to study the finitetime convergence of MAS. There are a lot of literature studies on the fast consensus problem of dynamic MAS, but few literatures devote to the studies on the finite-time group consensus problem. For example, the problem of finite-time formation control for MAS with general linear dynamics is investigated in [14]. Referring to [15], a novel finite-time containment control framework for MAS is established, which solves the containment control problem at any preset time with static or dynamic leaders. In [16], based on the aid of an auxiliary system, a new class of finite-time consensus protocols is proposed for the MAS by the aid of an auxiliary system. In [17], a distributed consensus protocol is presented to make the MAS locally or globally reach consensus in finite time. In [18], a nonfragile controller design protocol is proposed, which guarantees the finite-time stability of the resulting closed-loop system in the presence of time-varying delay in the input channel. Compared with the finite-time control in linear systems [14-18], the finite-time control in $[19,20]$ also applies to nonlinear multiagent systems. In [19], the finite-time consensus problem of heterogeneous MAS composed of both linear and nonlinear dynamic agents is investigated. The problem of finite-time consensus in probability for second-order stochastic MAS with nonidentical nonlinear dynamics is investigated in [20]. As far as the authors know, there are few reports on the study of finite-time group consensus of MAS.

Based on the above research results, we will focus on the finite-time group flocking of dynamic MAS in this paper. The main contributions of this paper are listed as follows:

(1) We propose a first-order group control algorithm, where cooperative relationships and competitive mechanisms are introduced into the model of MAS. Under the action of the control protocol, multiple agents converge to two equilibrium trajectories and achieve group consensus.

(2) We propose a second-order group control algorithm, which is a distributed nonlinear control protocol. With the help of the cooperation control, the group consensus of MAS is achieved, where the moving trajectories of dynamic agents will converge to two groups flocking.

(3) We study the cooperation control algorithm of the distributed network by applying Lyapunov stability and homogeneity theory and obtain the finite-time convergence conditions for group consensus of MAS in directed communication networks.
The rest of the paper is organized as follows. The preliminaries and problem formulations are stated in Section 2. The main results on finite-time group consensus of MAS are given in Section 3. The numerical simulations are provided in Section 4. Conclusions are presented in Section 5.

\section{Preliminaries}

In this section, some preliminaries on graph theory and problem formulations are given.

Let $G=(V, \omega, A)$ be a weighted directed graph with the set of nodes $V=\left\{v_{1}, v_{2}, \ldots, v_{n+m}\right\}$, the set of edges $\omega \subseteq V \times V$, and the nonsymmetrical weighted adjacency matrix $\bar{A}$ with real adjacency element $a_{i j}$. An edge of $G$ is denoted by $e_{i j}=\left(v_{i}, v_{j}\right)$, which starts from node $v_{i}$ and ends at node $v_{j}$. The adjacency elements associated with the edges of the graph are nonzero, i.e., $a_{i j} \neq 0$ if and only if $e_{i j} \in \omega$. For all nodes, it is assumed that $a_{i i}=0$. There are two ways to describe the communication connection between two nodes. If there is a communication link between $v_{i}$ and $v_{j}$, the sending and receiving of information can be represented by $v_{i} \longrightarrow v_{j}$ or $v_{j} \longrightarrow v_{i}$, respectively. In the neighbors' set, node $v_{i}$ is denoted by $N_{i}=\left\{v_{j} \mid \nu_{j} \in V:\left(\nu_{j}, v_{i}\right) \in \omega\right\}$. The Laplacian matrix $L(G)=\left[l_{i j}\right]_{(n+m) \times(n+m)}$ of topology $G$ is defined by

$$
l_{i j}= \begin{cases}-a_{i j}, & j \neq i, \\ \sum_{k=1, k \neq i}^{n+m} a_{i k}, & j=i .\end{cases}
$$

The in-degree and out-degree of node $i$ are defined as

$$
\begin{gathered}
\operatorname{Deg}_{\text {in }}(i)=\sum_{j=1}^{n+m} a_{i j}, \\
\operatorname{Deg}_{\text {out }}(i)=\sum_{j=1}^{n+m} a_{j i} .
\end{gathered}
$$

The matrix $D=\operatorname{diag}\left\{\sum_{j=1}^{n+m} a_{1 j}, \ldots, \sum_{j=1}^{n+m} a_{n+m, j}\right\}$ is defined as a degree matrix. The Laplacian matrix satisfies $L=D-A$. It is assumed that all agents communicate with each other through sensors; thus, the undirected network topology of MAS is constructed.

Definition 1. A graph $G_{1}=\left\{V_{1}, \omega_{1}, A_{1}\right\}$ is said to be a subgraph of a graph $G=\{V, \omega, A\}$ if $V_{1} \subseteq V$ and $\omega_{1} \subseteq \omega$.

Suppose a group $G=\{V, \omega, A\}$ can be partitioned into two subgraphs $G_{1}=\left\{V_{1}, \omega_{1}, A_{1}\right\}$ and $G_{2}=\left\{V_{2}, \omega_{2}, A_{2}\right\}$ with $V_{1} \cup V_{2}=V$ and $V_{1} \cap V_{2}=\Phi$. Without loss of generality, a network graph $G$ with $n+m(n, m>1)$ agents indexed by $1,2, \ldots, n+m$ is consisted with nodes $1,2, \ldots, n$ in subgraph $G_{1}$ and nodes $n+1, n+2, \ldots, n+m$ in subgraph $G_{2}$.

Assumption 1. There is a balance of effects between the subgraph $G_{1}$ and $G_{2}$ with $\sum_{j=n+1}^{n+m} a_{i j}=0, \forall i=G_{1}$, and $\sum_{j=1}^{n} a_{i j}=0, \forall i=G_{2}$.

Remark 1. The weighting factor $a_{i j}$ is permitted to be negative, which means that the effect is the competition mechanism. This provides additional flexibility in 
applications and makes the dynamic behaviors of agents more complex. Moreover, Assumption 1 means an in-degree balance of effect between the two groups.

Definition 2. Considering the dynamics of distributed MAS in the graph $G=G_{1} \cup G_{2}$, the cooperation control protocol is said to solve asymptotically a group consensus problem if for any initial state $x(0) \in R^{n}$ if the states $x=$ $\left[\begin{array}{llll}x_{1} & x_{2} & \ldots & x_{n+m}\end{array}\right]^{\mathrm{T}}$ satisfy

$$
\begin{aligned}
\text { (I) } \lim _{t \rightarrow \infty}\left\|x_{i}(t)-x_{j}(t)\right\|=0, & \forall i, j \in G_{1} \\
\text { (II) } \lim _{t \rightarrow \infty}\left\|x_{i}(t)-x_{j}(t)\right\|=0, & \forall i, j \in G_{2}
\end{aligned}
$$

Definition 3. Consider a nonlinear system in the form of

$$
\dot{x}=f(x), \quad \text { for } x \in R^{n},
$$

where $f: U_{0} \longrightarrow R^{n}$ is continuous on an open neighborhood $U_{0}$ of the origin $x=0$. The state of the system is finitetime convergent to its origin $x=0$ if for any initial condition $x_{0} \in U$, there exists a convergence time $T>0$, which is dependent on $x_{0}$ such that every solution $x\left(t, x_{0}\right)$ satisfies $\lim _{x \rightarrow T\left(x_{0}\right)} x\left(t, x_{0}\right)=0$ and $x\left(t, x_{0}\right)=0$ for $t \geq T\left(x_{0}\right)$. Moreover, if the origin $x=0$ is asymptotically stable and finitetime convergent in a neighborhood $U \subseteq U_{0} \subset R^{n}$, then the origin $x=0$ of the system is (locally) finite-time stable. If $U=R^{n}$, it is globally finite-time stable.

Lemma 1. Consider the nonlinear system described by equation (3). Suppose that there is a continuously differentiable function $V(x): U \longrightarrow R$ with real numbers $c>0$, $\alpha \in(0,1)$ and an open neighborhood $U_{0} \subset U$ of the origin such that $V(x)$ is positive definite on $U_{0}$ and that $\dot{V}(x)+c V^{\alpha} \leq 0, x_{0} \in U$. Then, the origin of system (3) is $f i$ nite-time stable. Moreover, if $T$ is the convergence time, then $T(x(0)) \leq\left(V(0)^{1-\alpha} / c(1-\alpha)\right)$ for all $x(0)$ in some open neighborhood of the origin. If $U=U_{0}=R^{n}$, the origin of system (3) is globally finite-time stable.

Definition 4. Consider a nonlinear system in the form of

$$
\begin{aligned}
\dot{x} & =f(x), \\
f(0) & =0, \\
x(0) & =x_{0},
\end{aligned}
$$

where $f(x)=\left[f_{1}(x), f_{2}(x), \ldots, f_{n}(x)\right]^{\mathrm{T}}$. If for any $\varepsilon>0$, $x \in R^{n}$, there exists $\left(r_{1}, r_{2}, \ldots, r_{n}\right)$, where $r_{i}>0$ such that $f(x)$ is satisfied.

$$
f_{i}\left(\varepsilon^{r_{1}} x_{1}, \varepsilon^{r_{2}} x_{2}, \ldots, \varepsilon^{r_{n}} x_{n}\right)=\varepsilon^{\kappa+r_{i}} f_{i}(x), \quad i=1,2, \ldots, n .
$$

Then, $f(x)$ has a degree of homogeneity $\kappa \in R$ with respect to $\left(r_{1}, r_{2}, \ldots, r_{n}\right)$, where $\left(r_{1}, r_{2}, \ldots, r_{n}\right)$ are called the extension coefficients.

Lemma 2. If $\dot{x}=f(x)$ is asymptotically stable at the equilibrium point and has the degree of homogeneity $\kappa<0$ with extension coefficients $\left(r_{1}, r_{2}, \ldots, r_{n}\right)$, the system is finite-time stable.

\section{Finite-Time Group Consensus of MAS}

3.1. Group Flocking Motion of First-Order MAS. It is assumed that networked systems are composed of two subgraphs with $n+m$ agents randomly connected, where $G_{1}=\{1,2, \ldots, n\}$ and $G_{2}=\{n+1, n+2, \ldots, n+m\}$. The following dynamics of the first-order MAS is considered:

$$
\dot{x}_{i}(t)=u_{i}(t), \quad i \in G,
$$

where $x_{i} \in R$ is the position state and $u_{i} \in R$ is the control protocol. In order to realize the group consensus of MAS in finite time, the control protocol is designed as

$$
u_{i}(t)= \begin{cases}\operatorname{sig}\left\{\sum_{j \in N_{1 i}} a_{i j}\left(x_{j}-x_{i}\right)+b_{i}\left(x_{01}-x_{i}\right)+\sum_{j \in N_{2 i}} a_{i j}\left(x_{j}-x_{i}\right)\right\}^{\alpha}, & \forall i \in G_{1}, \\ \operatorname{sig}\left\{\sum_{j \in N_{1 i}} a_{i j}\left(x_{j}-x_{i}\right)+\sum_{j \in N_{2 i}} a_{i j}\left(x_{j}-x_{i}\right)+b_{i}\left(x_{02}-x_{i}\right)\right\}^{\alpha}, & \forall i \in G_{2},\end{cases}
$$

where $a_{i j} \in R$ with $a_{i j}>0, \forall i, j \in G_{1}$ or $\forall i, j \in G_{2}$. The set $N_{1 i}=\left\{v_{j} \in V_{1}:\left(v_{i}, v_{j}\right) \in \omega\right\}$ and $N_{2 i}=\left\{v_{j} \in V_{2}:\left(v_{i}, v_{j}\right) \in\right.$ $\omega\}$ is the neighbor set of agent $i$. The variables $x_{01}$ and $x_{02}$ are the states of the leaders with dynamics $\dot{x}_{0 k}(t)=0, \quad k=1,2$. If followers $i$ can receive information from the leader $x_{0 k}$, then $b_{i}>0$; otherwise, $b_{i}=0$.

Assumption 2. There are two leader-agents in the graph, and the leader is a globally reachable node in every subgraph, respectively.
Theorem 1. Considering the formation of first-order $d y$ namics (6), it is assumed that the network topology is composed of $n+m$ agents with two subgraphs. If Assumptions 1 and 2 are established for dynamic system (6) with control protocol (7), the finite-time group consensus can be realized for the MAS.

Proof. Let $\bar{x}_{i}(t)=x_{i}(t)-x_{0 k}(t)$, then dynamic system (6) with control protocol (7) is written as

$$
\dot{\bar{x}}(t)=\operatorname{sig}[-H \bar{x}(t)]^{\alpha}
$$


where $\bar{x}(t)=\left[\bar{x}_{1}(t), \bar{x}_{2}(t), \ldots, \bar{x}_{n+m}(t)\right]^{\mathrm{T}}, H=L(G)+B=$ $\left[\begin{array}{cc}L\left(G_{1}\right)+B_{1} & A_{1}(t) \\ A_{2}(t) & L\left(G_{2}\right)+B_{2}\end{array}\right]$

$A_{1}=-\left[\begin{array}{cccc}a_{1, n+1}(t) & a_{1, n+2}(t) & \cdots & a_{1, n+m}(t) \\ a_{2, n+1}(t) & a_{2, n+2}(t) & \cdots & a_{2, n+m}(t) \\ \vdots & \vdots & \ddots & \vdots \\ a_{n, n+1}(t) & a_{n, n+2}(t) & \cdots & a_{n, n+m}(t)\end{array}\right]$,

$A_{2}=-\left[\begin{array}{cccc}a_{n+1,1}(t) & a_{n+1,2}(t) & \cdots & a_{n+1, n}(t) \\ a_{n+2,1}(t) & a_{n+2,2}(t) & \cdots & a_{n+2, n}(t) \\ \vdots & \vdots & \ddots & \vdots \\ a_{n+m, 1}(t) & a_{n+m, 2}(t) & \cdots & a_{n+m, n}(t)\end{array}\right]$,

$B=\operatorname{diag}\left\{b_{i}, \quad i=1, \ldots, n+m\right\}, B_{1}=\operatorname{diag}\left\{b_{i}, \quad i=1, \ldots, n\right\}$, and $B_{2}=\operatorname{diag}\left\{b_{i}, \quad i=n+1, \ldots, n+m\right\}$. With Assumptions 1 and 2 , the matrix $H$ is positive definite.

Let $y=-H \bar{x}(t)$; it can be obtained $\dot{\bar{x}}(t)=\operatorname{sig}[y]^{\alpha}$ from equation (8). Let the Lyapunov function be $V=(1 / 2) \bar{x}^{\mathrm{T}} H \bar{x}$; thus, the derivative of the Lyapunov function is

$$
\begin{aligned}
\dot{V} & =\bar{x}^{\mathrm{T}} H \dot{\bar{x}} \\
& =-y \operatorname{sig}(y)^{\alpha} \\
& =-\left(y_{1}, \ldots, y_{n+m}\right)\left(\begin{array}{c}
\left|y_{1}\right|^{\alpha} \operatorname{sign}\left(y_{1}\right) \\
\vdots \\
\left|y_{n+m}\right|^{\alpha} \operatorname{sign}\left(y_{n+m}\right)
\end{array}\right) \\
& =-\left(\left|y_{1}\right|^{\alpha+1}+\cdots+\left|y_{n+m}\right|^{\alpha+1}\right) \\
& =-\left[\sum_{k=1}^{n+m}\left|y_{k}\right|^{\alpha+1}\right] \leq-\left[\sum_{k=1}^{n+m}\left(y_{k}\right)^{2}\right]^{(\alpha+1 / 2)} .
\end{aligned}
$$

Since

$\sum_{k=1}^{n+m}\left(y_{k}\right)^{2}=y^{\mathrm{T}} y=\bar{x}^{\mathrm{T}} H^{\mathrm{T}} H \bar{x} \geq 2 \lambda_{\text {min }}\left((1 / 2) \bar{x}^{\mathrm{T}} H \bar{x}\right)$, where $\lambda_{\text {min }}$ is a nonzero minimum eigenvalue of the matrix $H$, it has

$$
\dot{V} \leq-\left[2 \lambda_{\min }\right]^{(\alpha+1 / 2)} V^{(\alpha+1 / 2)}
$$

Based on Lemma 1, system (6) is asymptotically stable in finite time at the equilibrium point, and the finite time $T[x(0)]$ satisfies

$$
T_{0}=T[x(0)] \leq \frac{2 V(0)^{(1-\alpha / 2)}}{(1-\alpha)\left(2 \lambda_{\min }\right)^{(\alpha+1 / 2)}} .
$$

Therefore, the fast convergence of the group flocking motion of first-order MAS can be realized.

3.2. Group Flocking Motion of Second-Order MAS. In this section, the group flocking of second-order MAS is studied. The dynamic system of agents is considered:

$$
\begin{aligned}
& \left\{\begin{array}{l}
\dot{x}_{i}(t)=v_{i}(t), \\
\dot{v}_{i}(t)=u_{i}(t),
\end{array}\right. \\
& i \in\{1,2, \ldots, n+m\} .
\end{aligned}
$$

Assume there are two leader-agents in the graph and the leader is a globally reachable node in every subgraph, respectively. The dynamics of the leader is

$$
\begin{aligned}
& \left\{\begin{array}{l}
\dot{x}_{0 k}(t)=v_{0 k}(t), \\
\dot{v}_{0 k}(t)=0
\end{array}\right. \\
& k=1,2 \text {, }
\end{aligned}
$$

where $x_{0 k}$ is the position of the leader and $v_{0 k}$ is the velocity of the leader.

In order to realize the group flocking convergence of the second-order MAS in finite time, the control protocol is designed as

$$
u_{i}(t)=\left\{\begin{array}{l}
\operatorname{sig}\left\{\left[\sum_{j \in N_{1 i}} a_{i j}\left(x_{j}-x_{i}\right)+b_{i}\left(x_{01}-x_{i}\right)\right]+\sum_{j \in N_{2 i}} a_{i j}\left(x_{j}-x_{i}\right)\right\}^{\alpha_{1}}+\operatorname{sig}\left\{\left[\sum_{j \in N_{1 i}} a_{i j}\left(v_{j}-v_{i}\right)+b_{i}\left(v_{01}-v_{i}\right)\right]+\sum_{j \in N_{2 i}} a_{i j}\left(v_{j}-v_{i}\right)\right\}^{\alpha_{2}}, \quad \forall i \in G_{1}, \\
\operatorname{sig}\left\{\sum_{j \in N_{1 i}} a_{i j}\left(x_{j}-x_{i}\right)+\left[\sum_{j \in N_{2 i}} a_{i j}\left(x_{j}-x_{i}\right)+b_{i}\left(x_{02}-x_{i}\right)\right]\right\}^{\alpha_{1}}+\operatorname{sig}\left\{\sum_{j \in N_{1 i}} a_{i j}\left(v_{j}-x_{i}\right)+\left[\sum_{j \in N_{2 i}} a_{i j}\left(v_{j}-v_{i}\right)+b_{i}\left(v_{02}-v_{i}\right)\right]\right\}^{\alpha_{2}}, \quad \forall i \in G_{2},
\end{array}\right.
$$

where $0<\alpha_{1}<1$ and $\alpha_{2}=\left(2 \alpha_{1} / \alpha_{1}+1\right)$.

Remark 2. Compared with the first-order MAS, the leader of the second-order MAS has the velocity with the acceleration zero, which means that if other agents receive information from the leader, they will adjust their speed under control protocol (14), eventually catch up the speed consistent with the leader.

Remark 3. In the control protocol of first-order/second-order MAS, the weight value $b_{i}$ could be zero, which means that there is the leaderless condition of the dynamic MAS or the agents do not receive information from the leader.
Theorem 2. Considering the formation of second-order dynamic multiagent system (12), it is assumed that the network topology is composed of $n+m$ agents with two subgraphs. If Assumptions 1 and 2 are established for dynamic system (12) with control protocol (14), the finite-time group consensus can be realized for second-order MAS.

Proof. Let $\bar{x}_{i}(t)=x_{i}(t)-x_{0 k}(t)$ and $\bar{v}_{i}(t)=v_{i}(t)-v_{0 k}(t)$. Dynamic system (12) with control protocol (14) is written as

$$
\dot{\bar{v}}=\operatorname{sig}(-H \bar{x})^{\alpha_{1}}+\operatorname{sig}(-H \bar{v})^{\alpha_{2}},
$$


where $\bar{x}(t)=\left[\bar{x}_{1}(t), \bar{x}_{2}(t), \ldots, \bar{x}_{n+m}(t)\right]^{\mathrm{T}}$ and $\bar{v}(t)=\left[\bar{v}_{1}(t)\right.$, $\left.\bar{v}_{2}(t), \ldots, \bar{v}_{n+m}(t)\right]^{\mathrm{T}}$. Let $z=-H \bar{x}$ and $w=-H \bar{v}$. We definite the Lyapunov function $Q=Q_{1}+Q_{2}$, where

$$
\begin{aligned}
& Q_{1}=\bar{v}^{\mathrm{T}} H \bar{v}, \\
& Q_{2}=\frac{2|z|^{\alpha_{1}+1}}{\alpha_{1}+1} .
\end{aligned}
$$

The derivative of the Lyapunov function along system (12) is

$$
\begin{aligned}
\dot{Q}_{1} & =2 \bar{v}^{\mathrm{T}} H \dot{\bar{v}} \\
& =-2 w^{\mathrm{T}}\left[\operatorname{sig}(z)^{\alpha_{1}}+\operatorname{sig}(w)^{\alpha_{2}}\right] \\
& =-2 w^{\mathrm{T}} \operatorname{sig}(z)^{\alpha_{1}}-2 w^{\mathrm{T}} \operatorname{sig}(w)^{\alpha_{2}} \\
\dot{Q}_{2} & =2 \operatorname{sig}(z)^{\alpha_{1}} \cdot \dot{z}=2 \operatorname{sig}(z)^{\alpha_{1}} \cdot w .
\end{aligned}
$$

It can be obtained

$$
\begin{aligned}
\dot{Q} & =\dot{Q}_{1}+\dot{Q}_{2}=-2 w^{\mathrm{T}} \operatorname{sig}(w)^{\alpha_{2}} \\
& =-2|w|^{\alpha_{2}+1} \leq 0 .
\end{aligned}
$$

Note that the equilibrium states of the system satisfy $\dot{\bar{x}}=$ 0 and $\dot{\bar{v}}=0$. We can obtain that $\bar{v}=0$ and $u(t)=0$. That is,

$$
\operatorname{sig}(-H \bar{x})^{\alpha_{1}}=0 .
$$

We can obtain the equilibrium states of the system which satisfy $\bar{x}=0$ and $\bar{v}=0$.

When $\dot{Q} \equiv 0$, there is $w=0$. We can obtain $\bar{v}=0$ and $\dot{\bar{v}}=0$ from $w=-H \bar{v}$. It has $\bar{x}=0$ and $\bar{v}=0$. That is, when $\dot{Q} \equiv 0$, there is only the equilibrium point in this solution set. According to the principle of LaSalle's invariant set, the system is asymptotically stable at the equilibrium point. It has $\lim _{t \rightarrow \infty} \bar{x}_{i}(t)=\lim _{t \longrightarrow \infty}\left(x_{i}(t)-x_{0 k}(t)\right)=0$ and $\lim _{t \rightarrow \infty} \bar{v}_{i}(t)=$ $\lim _{t \rightarrow \infty}\left(v_{i}(t)-v_{0 k}(t)\right)=0$

For second-order MAS (12), it is assumed that

$$
\left\{\begin{array}{l}
f_{1}\left(x_{i}, v_{i}\right)=v_{i}(t) \\
f_{2}\left(x_{i}, v_{i}\right)=u_{i}(t)
\end{array}\right.
$$

Let $\gamma_{1}=2, \gamma_{2}=\alpha_{1}+1$, and $\kappa=\alpha_{1}-1$. We can obtain from Lemma 2 that

$$
\begin{aligned}
f_{1}\left(\varepsilon^{\gamma_{1}} x_{i}, \varepsilon^{\gamma_{2}} v_{i}\right)= & \varepsilon^{\gamma_{2}} v_{i}=\varepsilon^{\gamma_{1}+\kappa} f_{1}\left(x_{i}, v_{i}\right), \\
f_{2}\left(\varepsilon^{\gamma_{1}} x_{i}, \varepsilon^{\gamma_{2}} v_{i}\right)= & \varepsilon^{\gamma_{1} \alpha_{1}} \operatorname{sig}\left\{\left[\sum_{j \in N_{1 i}} a_{i j}\left(x_{j}-x_{i}\right)+b_{i j}\left(x_{01}-x_{i}\right)\right]+\sum_{j \in N_{2 i}} a_{i j}\left(x_{j}+x_{i}\right)\right\}^{\alpha_{1}} \\
& +\varepsilon^{\gamma_{2} \alpha_{2}} \operatorname{sig}\left\{\left[\sum_{j \in N_{1 i}} a_{i j}\left(v_{j}-v_{i}\right)+b_{i j}\left(v_{01}-v_{i}\right)\right]+\sum_{j \in N_{2 i}} a_{i j}\left(v_{j}+v_{i}\right)\right\}^{\alpha_{2}} \\
& +\varepsilon^{\gamma_{1} \alpha_{1}} \operatorname{sig}\left\{\sum_{j \in N_{1 i}} a_{i j}\left(x_{j}+x_{i}\right)+\left[\sum_{j \in N_{2 i}} a_{i j}\left(x_{j}-x_{i}\right)+b_{i j}\left(x_{02}-x_{i}\right)\right]\right\}^{\alpha_{1}} \\
& +\varepsilon^{\gamma_{2} \alpha_{2}} \operatorname{sig}\left\{\sum_{j \in N_{1 i}} a_{i j}\left(v_{j}+x_{i}\right)+\left[\sum_{j \in N_{2 i}} a_{i j}\left(v_{j}-v_{i}\right)+b_{i j}\left(v_{02}-v_{i}\right)\right]\right\}^{\alpha_{2}} \\
= & \varepsilon^{\kappa+\gamma_{2}} f_{2}\left(x_{i}, v_{i}\right) .
\end{aligned}
$$

Therefore, multiagent dynamic system (12) with group flocking protocol (14) has the extension coefficient $(\underbrace{2,2, \ldots, 2}_{n+m}, \underbrace{\alpha_{1}+1, \alpha_{1}+1, \ldots, \alpha_{1}+1}_{n+m})$ and the degree of homogeneity $\kappa=\alpha_{1}-1<0$. According to Lemma 2 , the system can achieve the convergence in finite time.

Remark 4. When there are leaderless or other agents in the system who do not receive the leader's information, that is $b_{i}=0$, system (12) could still achieve the finite-time group flocking through control protocol (14) if there is a globally reachable node in each subgraph.

\section{Numerical Simulation}

It is assumed that the topology of MAS with two subgroups is shown in Figure 1. There are 9 agents in this topology graph, where agent 1-agent 7 are followers and agent L1 and agent $\mathrm{L} 2$ are the corresponding leaders in the two subgroups. The line between each agent indicates that the connected agents have information interaction, and the numbers indicate the associated weight.

4.1. Simulation of Finite-Time Convergence of First-Order MAS. It is assumed that the initial states of agents are $x(0)=$ $[5,8,6,12,4,2,-3]^{\mathrm{T}}$ and the initial states of leaders $x_{01}=2$ and $x_{02}=3$, respectively. Dynamic equation (6) and finite-time control protocol (7) are applied in the simulation with the parameter $\alpha=0.8$. The simulation results are shown in Figure 2 .

It can be seen that the positions of each agent finally converge to two equilibrium states with control protocol (7) in finite time $T=15 \mathrm{~s}$. The followers track the positions of leaders into two subgroups, and the group flocking motion of first-order MAS is realized in finite time. 


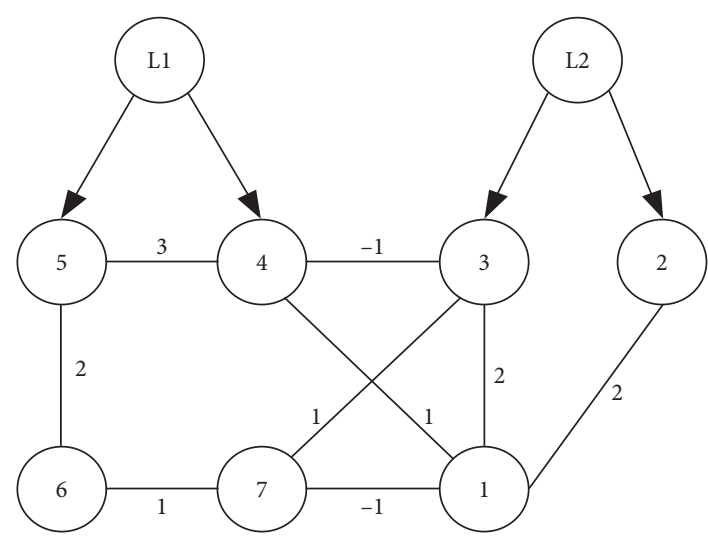

FIGURE 1: Topology of multiagent systems with leaders.

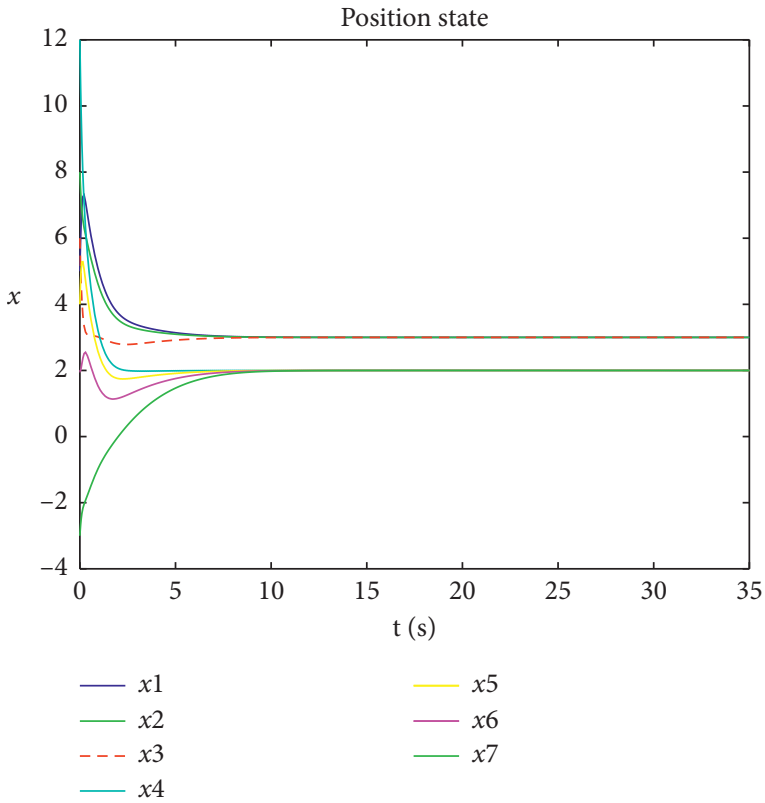

FIgURE 2: Motion trajectory of first-order MAS with leaders.

4.2. Simulation of Finite-Time Convergence of Second-Order MAS. It is assumed that the initial states and the initial speeds of agents are $x(0)=[5,8,6,12,4,2,5]^{\mathrm{T}}$ and $v(0)=[4,7,5,11,3,1,4]^{\mathrm{T}}$. Considering the leader in two subgraphs with the initial state and the initial speed, $x_{01}=$ $2, v_{01}=2$ and $x_{02}=3, v_{02}=3$, respectively. Dynamic equation (12) and finite-time control protocol (14) are applied in the simulation with the parameters $\alpha_{1}=0.8$ and $\alpha_{2}=$ $\left(2 \alpha_{1} / \alpha_{1}+1\right)$. The simulation results of the system motion are shown in Figure 3.

It can be seen that the motion trajectories of each agent finally converge to two equilibrium states with control protocol (14). The followers track the trajectory of leaders into two subgroups in finite time $T=15 \mathrm{~s}$, and the secondorder MAS realizes the group flocking motion in finite time.

4.3. Simulation of Finite-Time Convergence of MAS without Leaders. Now, we assume the topology graph of MAS without leaders which is shown in Figure 4. In the topology
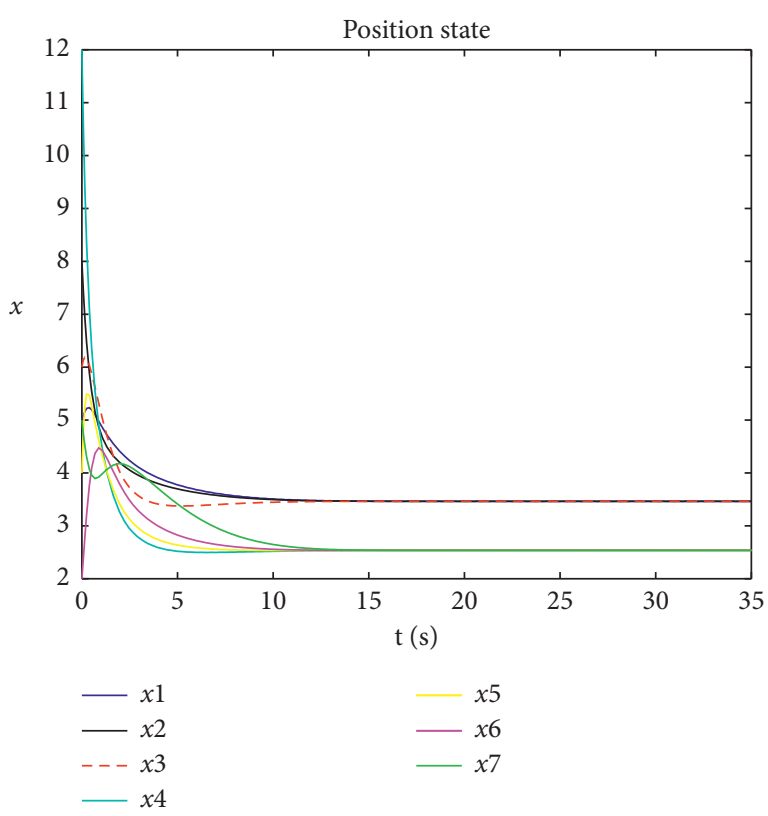

FIGURE 3: Motion trajectories of second-order MAS with leaders.

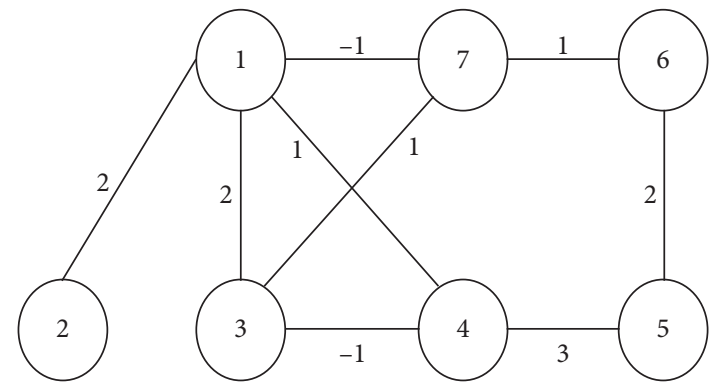

Figure 4: Topology of multiagent systems without leaders.

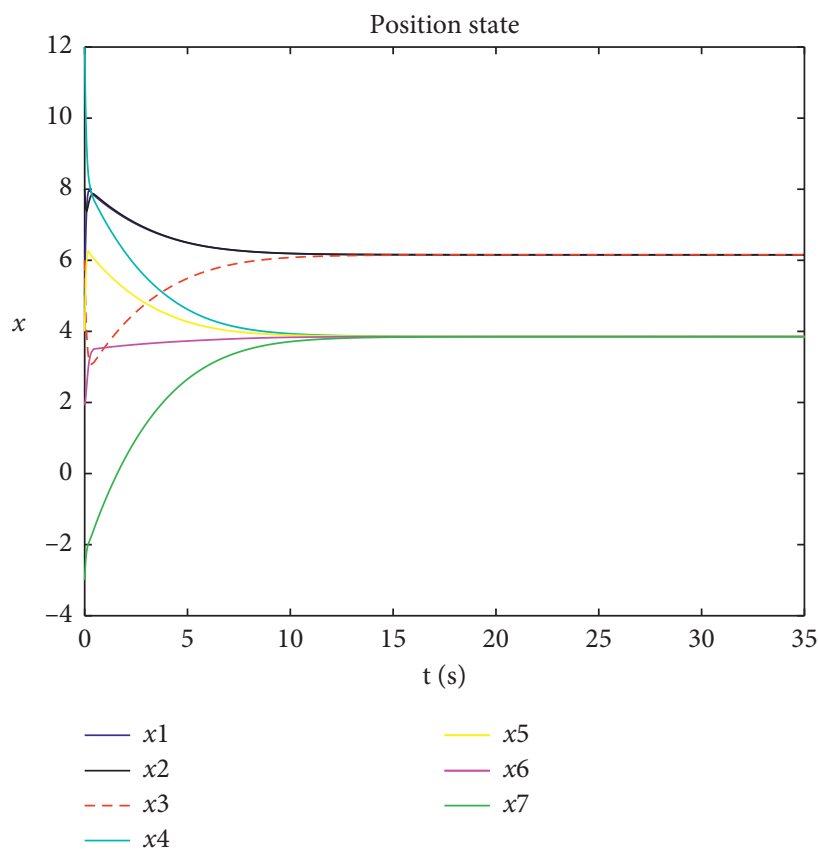

Figure 5: Motion trajectories of first-order MAS without leaders. 


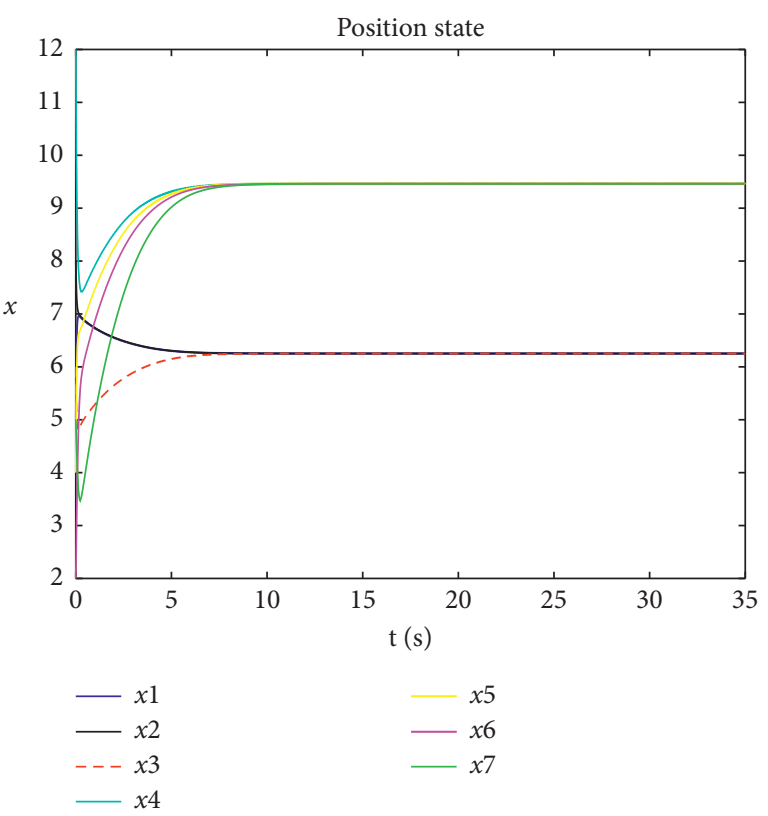

FIGURE 6: Motion trajectory of second-order MAS without leaders.

graph, agents 1-7 are all substantive agents. There is information interaction between each agent.

The initial values and the parameters of agents are the same as Sections 4.1 and 4.2. Dynamic system (6) with control protocol (7) is applied in the simulation; the motion trajectory of first-order MAS without leaders is shown in Figure 5. Dynamic system (12) with control protocol (14) is applied in the simulation; the motion trajectory of second-order MAS without leaders is shown in Figure 6. It can be seen that the movement consensus of MAS without leaders is realized in finite time. The agents move into two subgroups, and finite-time group consensus is achieved.

\section{Conclusion}

In this paper, the finite-time group consensus of the firstorder/second-order dynamic MAS with/without leaders is studied. Two cooperation control protocols of MAS are proposed. By applying finite-time convergence theory and algebraic graph theory, the group flocking motions of dynamic MAS are investigated, and the convergence conditions of finite-time group consensus are obtained. The effectiveness of the proposed control algorithms is validated by numerical simulations. In future work, the coordinated motion of MAS with external interferences will be analyzed.

\section{Data Availability}

No data were used to support this study.

\section{Conflicts of Interest}

The authors declare that they have no conflicts of interest.

\section{Acknowledgments}

This research was supported by the National Natural Science Foundation of China (under grant 61673200) and the Major Basic Research Project of the Natural Science Foundation of Shandong Province of China (ZR2018ZC0438).

\section{References}

[1] H.-Y. Yang, X.-L. Zhu, and S.-Y. Zhang, "Consensus of second-order delayed multi-agent systems with leader-following," European Journal of Control, vol. 16, no. 2, pp. 188-199, 2010.

[2] H. Yang, Z. Zhang, and S. Zhang, "Consensus of second-order multi-agent systems with exogenous disturbances," International Journal of Robust and Nonlinear Control, vol. 21, no. 9, pp. 945-956, 2010.

[3] Y. Cao and Y. Sun, "Consensus of discrete-time third-order multi-agent systems in directed networks," Neurocomputing, vol. 177, pp. 394-400, 2016.

[4] X. Ai, S. Song, and K. You, "Second-order consensus of multiagent systems under limited interaction ranges," Automatica, vol. 68 , pp. 329-333, 2016.

[5] W. Zou, C. K. Ahn, and Z. Xiang, "Leader-following consensus of second-order nonlinear multi-agent systems with unmodeled dynamics," Neurocomputing, vol. 322, pp. 120129, 2018.

[6] F. Wang, H. Yang, and Y. Yang, "Swarming movement of dynamical multi-agent systems with sampling control and time delays," Soft Computing, vol. 23, no. 2, pp. 707-714, 2018.

[7] H.-Y. Yang, Y. Yang, F. Han, M. Zhao, and L. Guo, "Containment control of heterogeneous fractional-order multiagent systems," Journal of the Franklin Institute, vol. 356, no. 2, pp. 752-765, 2019.

[8] B. Liu, H. Su, F. Jiang, Y. Gao, L. Liu, and J. Qian, "Group controllability of continuous-time multi-agent systems," IET Control Theory \& Applications, vol. 12, no. 11, pp. 1665-1671, 2018.

[9] Q. Cui, D. Xie, and F. Jiang, "Group consensus tracking control of second-order multi-agent systems with directed fixed topology," Neurocomputing, vol. 218, pp. 286-295, 2016.

[10] A. Hu, J. Cao, M. Hu, and L. Guo, "Event-triggered group consensus for multi-agent systems subject to input saturation," Journal of the Franklin Institute, vol. 355, no. 15, pp. 7384-7400, 2018.

[11] J. Yu and L. Wang, "Group consensus in multi-agent systems with switching topologies and communication delays," Systems \& Control Letters, vol. 59, no. 6, pp. 340-348, 2010.

[12] G. Wen, Y. Yu, Z. Peng, and H. Wang, "Dynamical group consensus of heterogenous multi-agent systems with input time delays," Neurocomputing, vol. 175, pp. 278-286, 2016.

[13] B.-R. An, G.-P. Liu, and C. Tan, "Group consensus control for networked multi-agent systems with communication delays," ISA Transactions, vol. 76, pp. 78-87, 2018.

[14] Y. Liu and Z. Geng, "Finite-time formation control for linear multi-agent systems: a motion planning approach," Systems \& Control Letters, vol. 85, pp. 54-60, 2015.

[15] H. Wang, C. Wang, and G. Xie, "Finite-time containment control of multi-agent systems with static or dynamic leaders," Neurocomputing, vol. 226, pp. 1-6, 2017.

[16] X. Liu, J. Cao, N. Jiang, G. Hao, and S. Wang, "Finite-time consensus of second-order multi-agent systems via auxiliary system approach," Journal of the Franklin Institute, vol. 353, no. 7, pp. 1479-1493, 2016. 
[17] P. Tong, S. Chen, and L. Wang, "Finite-time consensus of multi-agent systems with continuous time-varying interaction topology," Neurocomputing, vol. 284, pp. 187-193, 2018.

[18] R. Sakthivel, S. Kanakalakshmi, B. Kaviarasan, Y.-K. Ma, and A. Leelamani, "Finite-time consensus of input delayed multiagent systems via non-fragile controller subject to switching topology," Neurocomputing, vol. 325, pp. 225-233, 2019.

[19] Y.-K. Zhu, X.-P. Guan, and X.-Y. Luo, "Finite-time consensus of heterogeneous multi-agent systems with linear and nonlinear dynamics," Acta Automatica Sinica, vol. 40, no. 11, pp. 2618-2624, 2014.

[20] L. Zhao and Y. Jia, "Finite-time consensus for second-order stochastic multi-agent systems with nonlinear dynamics," Applied Mathematics and Computation, vol. 270, pp. 278-290, 2015. 